\title{
Probing the dilaton in central exclusive processes at LHC
}

\author{
V. P. Goncalves* and W. K. Sauten $\dagger^{*}$ \\ High and Medium Energy Group, Instituto de Física e Matemática, \\ Universidade Federal de Pelotas, Caixa Postal 354, CEP 96010-900, Pelotas, RS, Brazil
}

(Dated: October 26, 2018)

\begin{abstract}
The existence of a dilaton as a pseudo-Nambu-Goldstone boson in spontaneous breaking of scale symmetry is predicted in beyond standard model theories in which electroweak symmetry is broken via strongly coupled conformal dynamics. Such a particle is expected to have a mass below the conformal symmetry breaking scale $f$ and couplings to standard model particles similar to those of the SM Higgs boson. In this paper we estimate, for the first time, the dilaton production in exclusive processes considering Pomeron - Pomeron $(\mathbb{P} \mathbb{P})$ and photon - photon $(\gamma \gamma)$ interactions, which are characterized by two rapidity gaps and intact hadrons in the final state. Our results indicate that if the dilaton is massive $\left(M_{\chi} \geq 2 M_{W}\right)$, the study of dilaton production by $\mathbb{P} \mathbb{P}$ interactions in $p p$ collisions can be useful to determine its mass and the conformal energy scale.
\end{abstract}

PACS numbers: $14.80 . \mathrm{Va}, 14.80 . \mathrm{Bn}, 12.38 . \mathrm{Bx}$

Keywords: Central exclusive production, Dilaton, Higgs boson, Hadronic collisions

The prediction of the existence of new scalar particles is a characteristic of several candidate theories beyond the standard model (SM) (See e.g. Ref. [1]). One of these particles is the dilaton, denoted as $\chi$, which is predicted to appear as a pseudo-Nambu-Goldstone boson in spontaneous breaking of scale symmetry [2]. In the particular scenario in which electroweak symmetry is broken via strongly coupled conformal dynamics, a neutral dilaton is expected with a mass below the conformal symmetry breaking scale $f$ and couplings to standard model particles similar to those of the SM Higgs boson. The searching of the dilaton in inclusive proton - proton collisions at LHC energies motivated a lot of work, with special emphasis in the discrimination of the dilaton from the SM Higgs signals [3 9 ]. In particular, in Refs. [3, 4] the authors have derived regions of the mass $M_{\chi}$ and the conformal breaking scale of the dilaton allowed by constraints from Higgs searches at LEP and LHC. They find that for low values of $f$, the dilaton is already excluded by the LHC in a large portion of parameter space $\left(M_{\chi}-f\right)$ and that for large $f$ and large $M_{\chi}$ the dilaton is not excluded but could be discovered at LHC with more luminosity. In this paper we extend these previous studies for exclusive processes, in which the hadrons colliding remain intact after the interaction, losing only a small fraction of their initial energy and escaping the central detectors [10]. The signal would be a clear one with a dilaton tagged in the central region of the detector accompanied by regions of low hadronic activity, the so-called "rapidity gaps". In contrast to the inclusive production, which is characterized by large QCD activity and backgrounds which complicate the identification of a new physics signal, the exclusive production will be characterized by a clean topology associated to hadron - hadron interactions mediated by colorless exchanges. Our analysis is motivated by the studies performed in Refs. [11 14], which demonstrated that central exclusive processes are very sensitive to Beyond Standard Model contributions.

In this letter we will calculate the dilaton production considering Pomeron - Pomeron $(\mathbb{P} \mathbb{P})$ or photon - photon $(\gamma \gamma)$ interactions in $p p$ and $P b P b$ collisions at LHC energies. These processes are represented in Figs. 1 (a) and (b), respectively, and can be written in the form

$$
h_{1}+h_{2} \rightarrow h_{1} \otimes \chi \otimes h_{2},
$$

where $h_{i}$ is a proton or a nucleus and $\chi$ is the dilaton. The basic characteristic of these processes is the presence of two rapidity gaps $(\otimes)$ in the final state, separating the dilaton from the intact outgoing hadrons. Experimentally, these processes have a very clear signal in the absence of pile-up, with the presence of the final state $\chi$ and no other hadronic activity seen in the central detector. Moreover, the measurement of the outgoing hadrons with installation of forward hadron spectrometers can be useful to separate the exclusive events [10]. Such possibility is currently under discussion in the ATLAS and CMS Collaborations at LHC. For comparison with our predictions for the dilaton production, we update previous estimates of the SM Higgs in exclusive processes and analyse the possibility of distinguish between these states in these processes.

In what follows we present a brief review of the theoretical description of Pomeron - Pomeron $(\mathbb{P} \mathbb{P})$ or photon - photon $(\gamma \gamma)$ interactions in $p p$ and $P b P b$ collisions at LHC energies. For the central exclusive production of a

*Electronic address: barros@ufpel.edu.br

$\dagger$ Electronic address: werner.sauter@ufpel.edu.br 

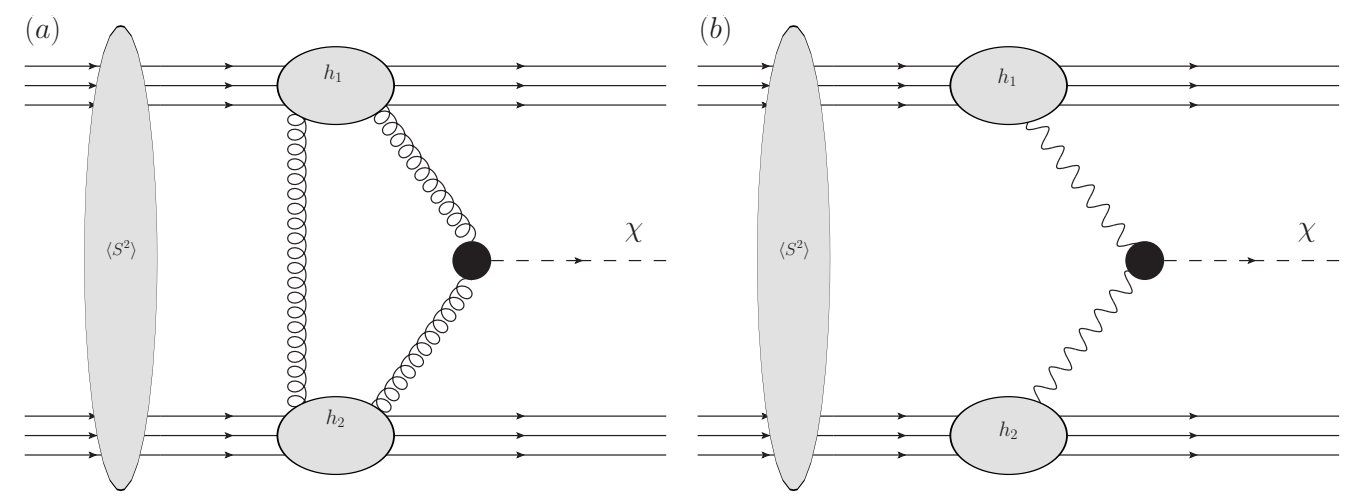

FIG. 1: Dilaton production in (a) Pomeron - Pomeron and (b) photon - photon interactions. $\left\langle\mathcal{S}^{2}\right\rangle$ is the gap survival probability, which gives the probability that secondaries, which are produced by soft rescatterings, do not populate the rapidity gaps.

dilaton by pomeron - pomeron interactions we consider the model proposed by Khoze, Martin and Ryskin [15 17 some years ago, denoted Durham model hereafter, which has been used to estimate a large number of different final states and have predictions in reasonable agreement with the observed rates for exclusive processes measured by the CDF collaboration [18 20] and in the Run I of the LHC (For a recent review see Ref. [21]). In this model, the total cross section for central exclusive production of a dilaton by $\mathbb{P} \mathbb{P}$ interactions can be expressed in a factorizated form as follows

$$
\sigma=\int d y\left\langle\mathcal{S}^{2}\right\rangle \mathcal{L}_{e x c l} \frac{2 \pi^{2}}{M_{\chi}^{3}} \Gamma(\chi \rightarrow g g)
$$

where $\left\langle\mathcal{S}^{2}\right\rangle$ is the gap survival probability (see below), $\Gamma$ stand for the partial decay width of the dilaton $\chi$ in a pair of gluons and $\mathcal{L}_{\text {excl }}$ is the effective luminosity, given by

$$
\mathcal{L}_{\text {excl }}=\left[\mathcal{C} \int \frac{d Q_{t}^{2}}{Q_{t}^{4}} f_{g}\left(x_{1}, x_{1}^{\prime}, Q_{t}^{2}, \mu^{2}\right) f_{g}\left(x_{2}, x_{2}^{\prime}, Q_{t}^{2}, \mu^{2}\right)\right]^{2}
$$

where $\mathcal{C}=\pi /\left[\left(N_{c}^{2}-1\right) b\right]$, with $b$ the $t$-slope $\left(b=4 \mathrm{GeV}^{-2}\right.$ in what follows $), Q_{t}^{2}$ is the virtuality of the soft gluon needed for color screening, $x_{1}$ and $x_{2}$ being the longitudinal momentum of the gluons which participate of the hard subprocess and $x_{1}^{\prime}$ and $x_{2}^{\prime}$ the longitudinal momenta of the spectator gluon. Moreover, the quantities $f_{g}$ are the skewed unintegrated gluon densities. Since

$$
\left(x^{\prime} \approx \frac{Q_{t}}{\sqrt{s}}\right) \ll\left(x \approx \frac{M_{\chi}}{\sqrt{s}}\right) \ll 1
$$

it is possible to express $f_{g}\left(x, x^{\prime}, Q_{t}^{2}, \mu^{2}\right)$, to single log accuracy, in terms of the conventional integrated gluon density $g(x)$, together with a known Sudakov suppression $T$ which ensures that the active gluons do not radiate in the evolution from $Q_{t}$ up to the hard scale $\mu \approx M_{\chi} / 2$. Following [16] we will assume that

$$
f_{g}\left(x, x^{\prime}, Q_{t}^{2}, \mu^{2}\right)=S_{g} \frac{\partial}{\partial \ln Q_{t}^{2}}\left[\sqrt{T\left(Q_{t}, \mu\right)} x g\left(x, Q_{t}^{2}\right)\right]
$$

where $S_{g}$ accounts for the single $\log Q^{2}$ skewed effect, being $S_{g} \sim 1.2(1.4)$ for LHC (Tevatron) (For a more detailed discussion about $S_{g}$ see Ref. [22]). The Sudakov factor $T\left(Q_{t}, \mu\right)$ is given by

$$
T\left(Q_{t}, \mu\right)=\exp \left\{-\int_{Q_{t}^{2}}^{\mu^{2}} \frac{d k_{t}^{2}}{k_{t}^{2}} \frac{\alpha_{s}\left(k_{t}^{2}\right)}{2 \pi} \int_{0}^{1-\Delta} d z\left[z P_{g g}(z)+\sum_{q} P_{q g}(z)\right]\right\}
$$

with $k_{t}$ being an intermediate scale between $Q_{t}$ and $\mu, \Delta=k_{t} /\left(\mu+k_{t}\right)$, and $P_{g g}(z)$ and $P_{q g}(z)$ are the leading order Dokshitzer - Gribov - Lipatov - Altarelli - Parisi (DGLAP) splitting functions [23]. In this paper we will calculate $f_{g}$ in the proton case considering that the integrated gluon distribution $x g\left(x, Q_{T}^{2}\right)$ is described by the 
MSTW parametrization [24]. In the nuclear case we will include the shadowing effects in $f_{g}^{A}$ considering that the nuclear gluon distribution is given by the EPS09 parametrization [25], where

$$
x g_{A}\left(x, Q_{T}^{2}\right)=A R_{g}^{A}\left(x, Q_{T}^{2}\right) x g_{p}\left(x, Q_{T}^{2}\right),
$$

with $R_{g}^{A}$ describing the nuclear effects in $x g_{A}$ and $A$ the number of mass of the nucleus. Moreover, the partial decay width of the dilaton into two gluons is given by [4]

$$
\Gamma_{\chi \rightarrow g g}\left(M_{\chi}\right)=\mathcal{C}_{g} \frac{v^{2}}{f^{2}} \frac{G_{F} \alpha_{s}^{2} M_{\chi}^{3}}{36 \sqrt{2} \pi^{3}}\left|\frac{3}{4} \sum_{f} F_{1 / 2}\left(\tau_{f}\right)\right|^{2}
$$

where $v=246 \mathrm{GeV}$ is the scale of electroweak symmetry breaking, $f$ is the energy scale of conformal scale (see discussion below), $G_{F}$ is the Fermi constant and $\alpha_{s}$ is the strong running coupling. The scaling variables are $\tau_{f}=$ $4 m_{f}^{2} / M_{\chi}^{2}, \tau_{W}=4 m_{W}^{2} / M_{\chi}^{2}$ and the sums runs over all fermions. The loop functions are given by the following expressions [26],

$$
\begin{aligned}
& F_{1}(\tau)=2+3 \tau+3 \tau(2-\tau) f(\tau) \\
& F_{1 / 2}(\tau)=-2 \tau[1+(1-\tau) f(\tau)]
\end{aligned}
$$

where

$$
f(\tau)= \begin{cases}{\left[\sin ^{-1}(1 / \sqrt{\tau})\right]^{2}} & , \tau \geq 1 \\ -\frac{1}{4}\left[\ln \left(\frac{1+\sqrt{1-\tau}}{1-\sqrt{1-\tau}}\right)-i \pi\right]^{2}, & \tau<1\end{cases}
$$

with $\tau=4 m_{i}^{2} / M_{\chi}^{2}$. Moreover, the coefficient $\mathcal{C}_{g}$ in Eq. (8) is given by

$$
\mathcal{C}_{g}=\frac{\left|-b_{G}+1 / 2 \sum_{i=q} F_{1 / 2}\left(\tau_{i}\right)\right|^{2}}{\left|1 / 2 \sum_{i=f} F_{1 / 2}\left(\tau^{2}\right)\right|^{2}}
$$

where $b_{G}=11-\frac{2}{3} n_{f}$, with $n_{f}=6$, and the sum runs over quarks only.

On the other hand, the cross section for the exclusive dilaton production in the two-photon fusion process, Fig. 1 (b), is given by [27]

$$
\sigma=\left\langle\mathcal{S}^{2}\right\rangle \int_{0}^{\infty} \frac{d \omega_{1}}{\omega_{1}} \int_{0}^{\infty} \frac{d \omega_{2}}{\omega_{2}} F\left(\omega_{1}, \omega_{2}\right) \hat{\sigma}_{\gamma \gamma \rightarrow \chi}\left(\omega_{1}, \omega_{2}\right)
$$

where $\hat{\sigma}_{\gamma \gamma \rightarrow \chi}$ is the cross section for the subprocess $\gamma \gamma \rightarrow \chi, \omega_{1}$ and $\omega_{2}$ the energy of the photons which participate of the hard process and $F$ is the folded spectra of the incoming particles (which corresponds to an "effective luminosity" of photons) which we assume to be given by [28]

$$
F\left(\omega_{1}, \omega_{2}\right)=2 \pi \int_{R_{A}}^{\infty} d b_{1} b_{1} \int_{R_{B}}^{\infty} d b_{2} b_{2} \int_{0}^{2 \pi} d \phi N_{1}\left(\omega_{1}, b_{1}\right) N_{2}\left(\omega_{2}, b_{2}\right) \Theta\left(b-R_{A}-R_{B}\right)
$$

where $b_{i}$ is the impact parameter of the hadrons in relation to the photon interaction point, $\phi$ is the angle between $\mathbf{b}_{1}$ and $\mathbf{b}_{2}, R_{i}$ are the projectile radii and $b^{2}=b_{1}^{2}+b_{2}^{2}-2 b_{1} b_{2} \cos \theta$. The theta function in Eq. (14) ensures that the hadrons do not overlap [28]. The Weizsäcker-Williams photon spectrum for a given impact parameter is given in terms of the nuclear charge form factor $F\left(k_{\perp}^{2}\right)$, where $k_{\perp}$ is the four-momentum of the quasi-real photon, as follows [29]

$$
N(\omega, b)=\frac{\alpha Z^{2}}{\pi^{2} \omega}\left|\int_{0}^{+\infty} d k_{\perp} k_{\perp}^{2} \frac{F\left(\left(\frac{\omega}{\gamma}\right)^{2}+\vec{b}^{2}\right)}{\left(\frac{\omega}{\gamma}\right)^{2}+\vec{b}^{2}} \cdot J_{1}\left(b k_{\perp}\right)\right|^{2}
$$

where $J_{1}$ is the Bessel function of the first kind. For a point-like nucleus one obtains that [29]

$$
N(\omega, b)=\frac{\alpha_{e m} Z^{2}}{\pi^{2}}\left(\frac{\xi}{b}\right)^{2}\left\{K_{1}^{2}(\xi)+\frac{1}{\gamma^{2}} K_{0}^{2}(\xi)\right\},
$$



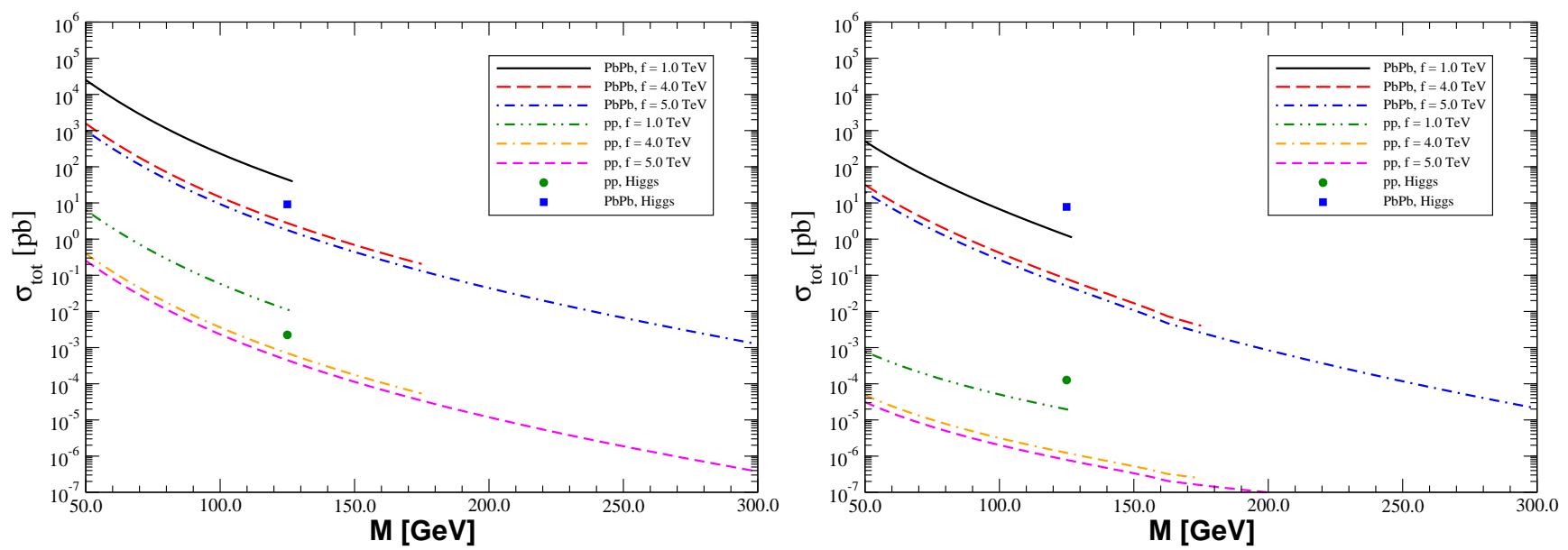

FIG. 2: (Color online) Total cross section for the dilaton production in (a) Pomeron - Pomeron and (b) photon - photon interactions in $p p$ and $\mathrm{PbPb}$ collisions as a function of the dilaton mass. The corresponding predictions for the SM Higgs production are also presented for comparison.

with $K_{0,1}$ being the modified Bessel function of second kind, $\xi=\omega b / \gamma v, v$ the velocity of the hadron, $\gamma$ the Lorentz factor and $\alpha_{e m}$ the electromagnetic coupling constant. This expression have been derived considering a semiclassical description of the electromagnetic interactions in peripheral collisions, which works very well for heavy ions (See e.g. [27]). For protons, it is more appropriate to obtain the equivalent photon spectrum from its elastic form factors in the dipole approximation (See e.g. [30]). An alternative is to use Eq. (16) assuming $R_{p}=0.7$ fm for the proton radius, which implies a good agreement with the parametrization of the luminosity obtained in [31] for proton-proton collisions. We will assume this procedure in what follows. The $\gamma \gamma \rightarrow \chi$ cross section can be expressed as follows

$$
\hat{\sigma}_{\gamma \gamma \rightarrow \chi}\left(\omega_{1}, \omega_{2}\right)=\int d s \delta\left(4 \omega_{1} \omega_{2}-s\right) \frac{8 \pi^{2}}{M_{\chi}} \Gamma_{\chi \rightarrow \gamma \gamma}\left(M_{\chi}\right) \delta\left(s-M_{\chi}^{2}\right)
$$

where partial decay width of the dilaton into two photons, $\Gamma_{\chi \rightarrow \gamma \gamma}$, was calculated in [4] and is given by:

$$
\Gamma_{\chi \rightarrow \gamma \gamma}\left(M_{\chi}\right)=\mathcal{C}_{\gamma} \frac{v^{2}}{f^{2}} \frac{G_{F} \alpha_{\mathrm{em}} M_{\chi}^{3}}{128 \sqrt{2} \pi^{3}}\left|F_{1}\left(\tau_{W}\right)+\sum_{f} N_{c} Q_{f}^{2} F_{1 / 2}\left(\tau_{f}\right)\right|^{2}
$$

where $\alpha_{\mathrm{em}}$ is the electromagnetic coupling constant, $N_{c}$ is the number of colors, $Q_{f}$ is the fermion charge. Moreover, the coefficient $\mathcal{C}_{\gamma}$ is given by [4]

$$
R_{\gamma}=\frac{\left|-b_{E M}+\sum_{i=f, b} N_{c, i} Q_{i}^{2} F_{i}\left(\tau_{i}\right)\right|^{2}}{\left|\sum_{i=f, b} N_{c, i} Q_{i}^{2} F_{i}\left(\tau_{i}\right)\right|^{2}}
$$

where $b_{E M}=-11 / 3$, the sum runs over fermions $(f)$ and bosons $(b), N_{c, i}$ is the color multiplicity number $\left(N_{c, i}=1\right.$ for bosons and leptons and $N_{c, i}=3$ for quarks) and $Q$ is the electric charge in units of $e$.

In what follows we will present our predictions for the dilaton production in $p p(\sqrt{s}=14 \mathrm{TeV})$ and $P b P b(\sqrt{s}=5.5$ $\mathrm{TeV}$ ) collisions at LHC energies. We assume $m_{W}=80.4 \mathrm{GeV}, m_{t}=173 \mathrm{GeV}, m_{b}=4.2 \mathrm{GeV}, m_{c}=1.4 \mathrm{GeV}$ and $m_{\tau}=1.77 \mathrm{GeV}$. Moreover, in order to obtain realistic predictions for the exclusive production of the dilaton, it is crucial to use an adequate value for the gap survival probability, $\left\langle\mathcal{S}^{2}\right\rangle$. This factor is the probability that secondaries, which are produced by soft rescatterings do not populate the rapidity gaps, and depends on the particles involved in the process and in the center-of-mass energy. For the case of the central exclusive production described by the Durham model we will assume that $\left\langle\mathcal{S}^{2}\right\rangle=3 \%$ for proton - proton collisions at LHC energies [16]. However, the value of the corresponding value of the survival probability for nuclear collisions still is an open question. Here we assume the conservative estimate proposed in Ref. [12] which assume that : $\left\langle\mathcal{S}^{2}\right\rangle_{A_{1} A_{2}}=\left\langle\mathcal{S}^{2}\right\rangle_{p p} /\left(A_{1} \cdot A_{2}\right)$. In contrast, for two-photon interactions, it is expected that the contribution of secondary interactions for the cross section will be negligible [10]. For simplicity, we will assume that $\left\langle\mathcal{S}^{2}\right\rangle_{A_{1} A_{2}}=\left\langle\mathcal{S}^{2}\right\rangle_{p p}=1$ for the dilaton production by two photons. However, this subject deserves a more detailed analysis. For our calculations of the SM Higgs production 

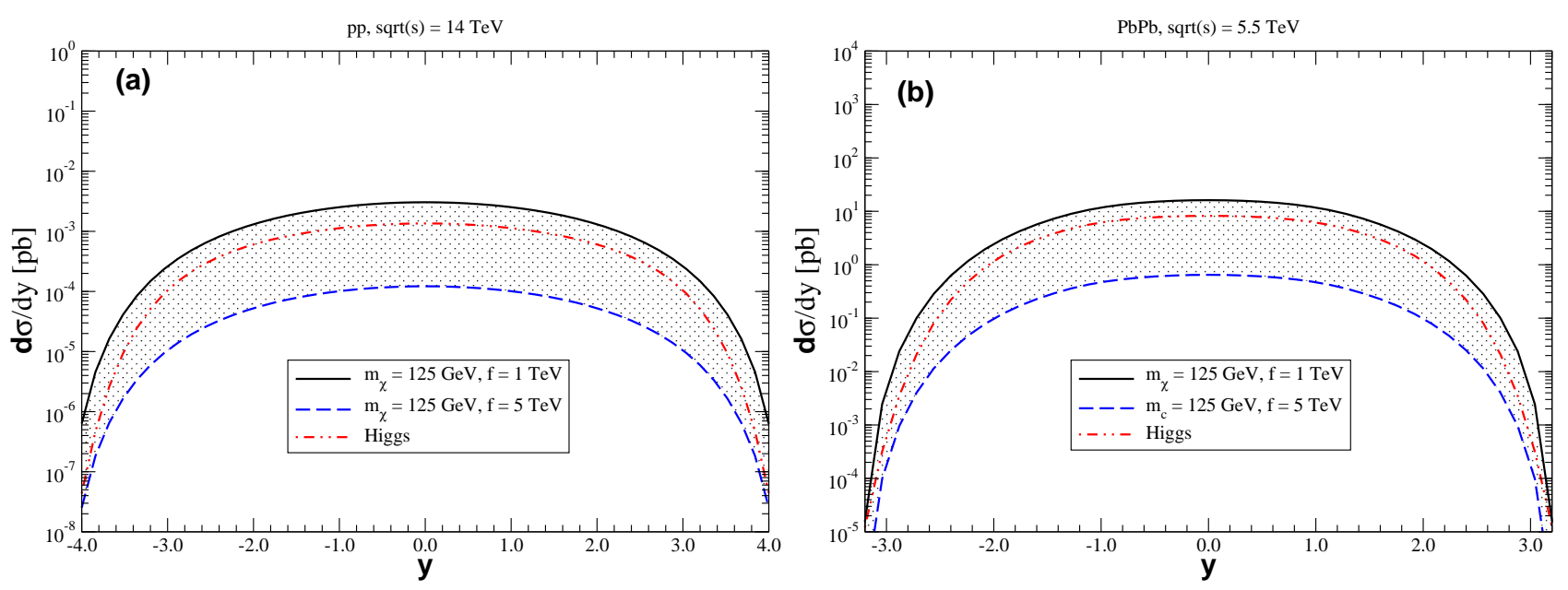

FIG. 3: (Color online) Rapidity distribution for the dilaton production in $\mathbb{P} \mathbb{P}$ interactions considering (a) $p p$ and (b) $P b P b$ collisions at LHC energies. The corresponding predictions for the SM Higgs production are also presented for comparison.

in exclusive processes, we will assume the same parameters described before and will take $\mathcal{C}_{\gamma / g} \frac{v^{2}}{f^{2}}=1$ in Eqs. (8) and (18). Moreover, we assume $M_{H}=125 \mathrm{GeV}$.

In Fig. 2 (a) and (b) we present our results for the dependence of the total cross sections on the mass of the dilaton considering $\mathbb{P} \mathbb{P}$ and $\gamma \gamma$ interactions in $p p$ and $P b P b$ collisions, respectively. For comparison, we also present the corresponding predictions for the SM Higgs production. Following previous studies [4, 5], which have derived allowed regions for the dilaton mass $M_{\chi}$ and conformal energy scale $f$ by considering the LHC data relevant to the double gauge boson decays of the dilaton, we restrict our analysis for the following ranges: (a) $f=1.0 \mathrm{TeV}$ and $M_{\chi} \leq 126$ GeV; (b) $f=4.0 \mathrm{TeV}$ and $M_{\chi} \leq 175 \mathrm{GeV}$ and (c) $f=5.0 \mathrm{TeV}$, with all values of $M_{\chi}$ being allowed [4, 5]. As the partial decay widths of the dilaton into gluons and photons, Eqs. (8) and (18), are proportional to $1 / f^{2}$, we obtain that the cross sections are strongly dependent on the choice of $f$, decreasing at larger values of the conformal energy scale. For the case of $\mathbb{P} \mathbb{P}$ interactions, Fig. 2 (a), we obtain that the predictions for the dilaton production in $\mathrm{PbPb}$ collisions is three orders of magnitude larger than for $p p$ collisions. In comparison with the SM Higgs predictions, we obtain that for $M_{\chi}=125 \mathrm{GeV}$ the dilaton cross section is larger than the exclusive SM Higgs cross section for $f=1.0$ $\mathrm{TeV}$ and a factor $\geq 2$ smaller for $f \geq 4.0 \mathrm{TeV}$. In contrast, for $\gamma \gamma$ interactions, Fig. 22(b), we obtain that at small $M_{\chi}$ the predictions for the dilaton production in $P b P b$ collisions is six orders of magnitude larger than for $p p$ collisions, which is directly associated to the $Z^{2}$ dependence of the nuclear photon flux. Moreover, we obtain that this difference decreases at larger values of $M_{\chi}$, which is associated to the fact that the maximum value of the photon energy in the photon flux is given by $\gamma / b_{\max }[29]$, where $\gamma$ is the Lorentz factor and $b_{\max }$ is proportional to the hadron radius. Consequently, the proton photon flux contains a larger number of energetic photons, which increases the cross section for the production of a massive final state in $p p$ collisions in comparison to the nuclear case. However, we predict very small values for the dilaton production induced by two-photon fusion in $p p$ cross section. Finally, in comparison to the SM Higgs cross section for $\gamma \gamma$ interactions, we obtain for $M_{\chi}=125 \mathrm{GeV}$ and $f=1.0 \mathrm{TeV}$ that the dilaton cross section is a factor five smaller than the SM Higgs one.

In Fig. 3 (a) and (b) we present our results for the rapidity distribution for the dilaton production in $\mathbb{P} \mathbb{P}$ interactions considering $p p$ and $\mathrm{PbPb}$ collisions at LHC energies, respectively. In these figures we assume $M_{\chi}=M_{H}=125 \mathrm{GeV}$. In agreement with the results presented in Fig. 2 (a), we obtain that the predictions are strongly dependent on $f$ and the dilaton production in nuclear collisions is four orders of magnitude than in $p p$ collisions. For $f=1 \mathrm{TeV}$, we predict larger values of the rapidity distribution for the dilaton production than the SM Higgs one.

In Table \we present our results for the production rates for dilaton production at LHC energies considering the $\mathbb{P} \mathbb{P}$ and $\gamma \gamma$ interactions assuming that $M_{\chi}=M_{H}=125 \mathrm{GeV}$. At LHC we assume the design luminosities $\mathcal{L}=10^{7} / 0.5$ $\mathrm{mb}^{-1} \mathrm{~s}^{-1}$ for $p p / P b P b$ collisions at $\sqrt{s}=14 / 5.5 \mathrm{TeV}$ and a run time of $10^{7}\left(10^{6}\right) \mathrm{s}$ for collisions with protons (ions). The predictions for the SM Higgs production also are presented for comparison. Due to the small luminosity for heavy ion collisions, we obtain very small values for the event rates of Higgs and dilaton production in $\mathbb{P} \mathbb{P}$ and $\gamma \gamma$ interactions. In contrast, we obtain that the event rates are a factor $\geq 10^{3}$ larger than for $p p$ collisions. For $\gamma \gamma$ interactions in $p p$ collisions we obtain that the predictions for the dilaton production $(f=1.0 \mathrm{TeV})$ are one order of magnitude smaller than the SM Higgs one, as expected from the Fig. 2. Moreover, the predictions for the dilaton and SM Higgs production in $\mathbb{P} \mathbb{P}$ interactions are a factor $\geq 10$ larger than those for $\gamma \gamma$ interactions. In particular, we 


\begin{tabular}{||c|c|c||}
\hline \hline $\mathbb{P P I}$ interactions & $p p$ collisions & $P b P b$ collisions \\
\hline \hline Dilaton & $1100(40)$ & $21.1 \times 10^{-3}\left(7.35 \times 10^{-4}\right)$ \\
\hline Higgs & 200 & $5 \times 10^{-3}$ \\
\hline \hline$\gamma \gamma$ interactions & $p p$ collisions & $P b P b$ collisions \\
\hline \hline Dilaton & $1.9\left(7.3 \times 10^{-2}\right)$ & $0.6 \times 10^{-3}\left(2.5 \times 10^{-5}\right)$ \\
\hline Higgs & 19 & $4.9 \times 10^{-3}$ \\
\hline \hline
\end{tabular}

TABLE I: Number of events by year for the production of a dilaton in $p p$ and $P b P b$ collisions considering $\mathbb{P} \mathbb{P}$ and $\gamma \gamma$ interactions. Values obtained for $M_{\chi}=125 \mathrm{GeV}$ and $f=1.0(5.0) \mathrm{TeV}$. The corresponding predictions for the SM Higgs production are also presented for comparison.

obtain that the dilaton production for $f=1.0 \mathrm{TeV}$ is a factor five larger than the SM Higgs one due to the magnitude of the factor $C_{g} v^{2} / f^{2}$ in Eq. (8), which is larger of one for this value of the conformal energy scale. At larger values of $f$, the Higgs production dominates due to the $1 / f^{2}$ dependence of the dilaton cross section.

Lets discuss now the potential backgrounds for the dilaton production in exclusive processes. The partial widths for the dilaton to decay into any SM final state were estimated in Refs. [4, 5]. Depending on the dilaton mass, different channels seem more favourable, and each of the decay channels has its own difficulties for the experimental identification. At $M_{\chi}<2 M_{W}$ the dilaton decay is characterized by a large $g g$ branching fraction, in contrast to the Higgs decay which is dominated by the decay in $b \bar{b}$ pair for $M_{H} \leq 140 \mathrm{GeV}$. This distinct behaviour is due to the enhancement of the $\chi g g$ coupling via the QCD beta function coefficient in Eq. (12). In contrast, for $M_{\chi}>2 M_{W}$ the dilaton decays predominantly to $W W, Z Z$ and $t \bar{t}$. Consequently, the main backgrounds are the $g g$ and $W W$ production in exclusive processes. The cross sections for these processes were estimated e.g. in Refs. [32, 33]. As demonstrated in Ref. [32], the $g g$ contribution dominates the central exclusive production of dijets. Comparing our results with those obtained in Ref. [32], we obtain that our predictions for the production of a dilaton with mass $M_{\chi}<140 \mathrm{GeV}$ which decays into a $g g$ final state is two orders of magnitude smaller than the exclusive $g g$ dijet production. Consequently, the identification of a light dilaton in exclusive processes considering this final state will be not possible. On the other hand, for the production of a massive dilaton which decays into a $W W$ pair, we need to compare our predictions with those presented in Ref. [33], which have demonstrated that the central exclusive $W^{+} W^{-}$production is dominated by the two - photon fusion. We obtain that also in this case the signal of the dilaton production will be smaller than the background, in particular for large $M_{\chi}$ masses, since the cross section for the $p p \rightarrow p p W^{+} W^{-}$(via $\left.\gamma \gamma\right)$ process is weakly dependent on the invariant mass $M_{W W}$ (See Fig. 14 in Ref. 33$]$ ). However, if the $\gamma \gamma$ and $\mathbb{P} \mathbb{P}$ processes are separated by measuring the four-momentum transfers squared in the proton lines, as planned for future studies at ATLAS and CMS, we have that the signal will be similar to the background associated to the $p p \rightarrow p p W^{+} W^{-}$(via $g g$ ) process. An alternative to search a massive dilaton in exclusive processes, is to consider its decay into a $Z Z$ pair and/or a Higgs pair. As demonstrated in Ref. [5], these two decay channels are similar to the $W W$ one for $M_{\chi}>200 \mathrm{GeV}$. The exclusive $Z Z$ production has been estimated in Ref. [14] as a probe of large extra dimension scenario. Comparing our results with the SM predictions presented in [14], we obtain that the signal will be larger than the background for large invariant masses of this final state. The magnitude of the double Higgs production in exclusive processes still is an open question, but it is expected to be very small. Consequently, the search of the dilaton considering its decay into a Higgs pair can also be a promising way.

Finally, lets summarize our main results and conclusions. In this paper we estimated, for the first time, the dilaton production in exclusive processes considering $\mathbb{P} \mathbb{P}$ and $\gamma \gamma$ interactions, which are characterized by two rapidity gaps and intact hadrons in the final state. Our goal was to verify if exclusive processes can be considered a viable alternative to the proposed searches of the dilaton in inclusive processes. In contrast to inclusive processes, where the incident hadrons dissociate and the final state is populated by a large number of particles, which makes the separation of the dilaton a hard task, in exclusive processes the incident hadron remains intact and the dilaton will be centrally produced, separated from the very forward hadrons by large rapidity gaps. Consequently, in exclusive processes the dilaton is expected to be produced in a clean environment. Moreover, if the momenta of the outgoing hadrons are measured by forward detectors, the mass of the dilaton is expected to be reconstructed with very precise resolution. All these aspects have motivated the analysis performed in this letter. We have considered one of the possible scenarios which predicts the dilaton and could be analysed at LHC, in which the scale invariance of the strong dynamics is manifest at very high energy but is spontaneously broken at a scale $f$, not too above the electroweak scale. Consequently, the identification of the dilaton provide a hint to the conformal nature of the strong sector. Taking into account the constraints in $f$ and $M_{\chi}$ from the Higgs searches at LEP and LHC, we have estimated the dilaton cross sections and event rates for the dilaton production induced by $\mathbb{P P}$ and $\gamma \gamma$ interactions in $p p$ and $P b P b$ collisions. For a dilaton with mass identical to the SM Higgs, we predict larger cross sections in comparison to the SM Higgs 
production in Pomeron - Pomeron interactions for $f=1.0 \mathrm{TeV}$, which is the minimal value of $f$ allowed for $M_{\chi}=125$ $\mathrm{GeV}$. At larger values of $f$, the dilaton cross section becomes smaller than the Higgs one due to its $1 / f^{2}$ dependence. In contrast, for photon - photon interactions, the dilaton cross section is smaller to the SM Higgs one for all allowed values of $f$. Taking into account the main decay channels of the dilaton, we have compared our predictions with potential backgrounds. Our results demonstrated that for a light dilaton the signal-to-background ratio will be very small, which implies that the probe of a light dilaton in exclusive processes at LHC will be not possible. In contrast, our results indicated that for a massive dilaton, which can be produced if $f>4.0 \mathrm{TeV}$, the signal-to-background ratio will be favourable if the dilaton decay channels into $Z Z$ and/or $H H$ are considered. Our main conclusion is that the study of exclusive processes can be useful to search a massive dilaton, as well as to constrain the mass and the conformal energy scale.

\section{Acknowledgements}

This work was partially financed by the Brazilian funding agencies FAPERGS, CNPq and CAPES.

[1] D. E. Morrissey, T. Plehn and T. M. P. Tait, Phys. Rept. 515, 1 (2012)

[2] T. E. Clark, C. N. Leung and S. T. Love, Phys. Rev. D 35, 997 (1987).

[3] V. Barger, M. Ishida and W. -Y. Keung, Phys. Rev. D 85, 015024 (2012)

[4] B. Coleppa, T. Gregoire and H. E. Logan, Phys. Rev. D 85, 055001 (2012)

[5] V. Barger, M. Ishida and W. -Y. Keung, Phys. Rev. Lett. 108, 101802 (2012)

[6] C. Coriano, L. Delle Rose, A. Quintavalle and M. Serino, JHEP 1306, 077 (2013)

[7] Z. Chacko, R. Franceschini and R. K. Mishra, JHEP 1304, 015 (2013)

[8] B. Bellazzini, C. Csaki, J. Hubisz, J. Serra and J. Terning, Eur. Phys. J. C 73, 2333 (2013)

[9] D. -W. Jung and P. Ko, arXiv:1401.5586 [hep-ph].

[10] M. G. Albrow, T. D. Coughlin and J. R. Forshaw, Prog. Part. Nucl. Phys. 65, 149 (2010).

[11] J. R. Ellis, J. S. Lee and A. Pilaftsis, Phys. Rev. D 71, 075007 (2005); M. Boonekamp, J. Cammin, S. Lavignac, R. B. Peschanski and C. Royon, Phys. Rev. D 73, 115011 (2006); P. J. Bussey, T. D. Coughlin, J. R. Forshaw and A. D. Pilkington, JHEP 0611, 027 (2006); V. A. Khoze, A. D. Martin, M. G. Ryskin and A. G. Shuvaev, Eur. Phys. J. C 68, 125 (2010); S. Heinemeyer, V. A. Khoze, M. G. Ryskin, M. Tasevsky and G. Weiglein, Eur. Phys. J. C 71, 1649 (2011); M. Tasevsky, Eur. Phys. J. C 73, no. 12, 2672 (2013)

[12] V. P. Goncalves and W. K. Sauter, Phys. Rev. D 82, 056009 (2010)

[13] P. Lebiedowicz, R. Pasechnik and A. Szczurek, Nucl. Phys. B 881, 288 (2014)

[14] V. P. Goncalves, W. K. Sauter and M. Thiel, Phys. Rev. D 89, 076003 (2014)

[15] V. A. Khoze, A. D. Martin and M. G. Ryskin, Eur. Phys. J. C 14, 525 (2000)

[16] V. A. Khoze, A. D. Martin and M. G. Ryskin, Eur. Phys. J. C 23, 311 (2002)

[17] V. A. Khoze, A. D. Martin and M. G. Ryskin, Eur. Phys. J. C 19, 477 (2001) [Erratum-ibid. C 20, 599 (2001)]

[18] T. Aaltonen et al. [CDF Collaboration], Phys. Rev. Lett. 99, 242002 (2007)

[19] T. Aaltonen et al. [CDF Collaboration], Phys. Rev. D 77, 052004 (2008)

[20] T. Aaltonen et al. [CDF Collaboration], Phys. Rev. Lett. 102, 242001 (2009)

[21] L. A. Harland-Lang, V. A. Khoze, M. G. Ryskin and W. J. Stirling, Int. Jour. Mod. Phys. A 29, 1430031 (2014)

[22] L. A. Harland-Lang, Phys. Rev. D 88, 034029 (2013)

[23] V. N. Gribov and L.N. Lipatov, Sov. J. Nucl. Phys 15, 438 (1972); Yu. L. Dokshitzer, Sov. Phys. JETP 46, 641 (1977); G. Altarelli and G. Parisi, Nucl. Phys. B126, 298 (1977).

[24] A. D. Martin, W. J. Stirling, R. S. Thorne and G. Watt, Eur. Phys. J. C 63, 189 (2009)

[25] K. J. Eskola, H. Paukkunen and C. A. Salgado, JHEP 0904, 065 (2009)

[26] J. F. Gunion, H. E. Haber, G. L. Kane and S. Dawson, Front. Phys. 80, 1 (2000).

[27] G. Baur, K. Hencken and D. Trautmann, J. Phys. G 24, 1657 (1998)

[28] G. Baur and L. G. Ferreira Filho, Nucl. Phys. A 518, 786 (1990).

[29] G. Baur, K. Hencken, D. Trautmann, S. Sadovsky, Y. Kharlov, Phys. Rep. 364, 359 (2002); C. A. Bertulani, S. R. Klein and J. Nystrand, Ann. Rev. Nucl. Part. Sci. 55, 271 (2005); K. Hencken et al., Phys. Rept. 458, 1 (2008).

[30] D. d'Enterria and J. P. Lansberg, Phys. Rev. D 81, 014004 (2010)

[31] J. Ohnemus, T. F. Walsh and P. M. Zerwas, Phys. Lett. B 328, 369 (1994)

[32] R. Maciula, R. Pasechnik and A. Szczurek, Phys. Rev. D 83, 114034 (2011); Phys. Rev. D 84, 114014 (2011)

[33] P. Lebiedowicz, R. Pasechnik and A. Szczurek, Nucl. Phys. B 867, 61 (2013) 